\title{
Possibility of one-stage surgery to reconstruct bone defects using the modified Masquelet technique with degradable calcium sulfate as a cement spacer: A case report and hypothesis
}

\author{
NAN JIANG ${ }^{1,2 *}$, CHENG-HE QIN ${ }^{1 *}$, YUN-FEI MA ${ }^{2}$, LEI WANG $^{1}$ and BIN YU ${ }^{1,2}$ \\ ${ }^{1}$ Department of Orthopaedics and Traumatology, ${ }^{2}$ Provincial Key Laboratory of Bone and \\ Cartilage Regenerative Medicine, Nanfang Hospital, Southern Medical University, Guangzhou 510515, P.R. China
}

Received November 11, 2015; Accepted January 21, 2016

DOI: $10.3892 /$ br. 2016.584

\begin{abstract}
In addition to autologous bone graft, vascularized fibular autograft and Ilizarov bone transfer, the Masquelet technique is another effective method to reconstruct bone defects. This technique was initially proposed in 1986 and consists of two stages. At the first stage, radical debridement is required and subsequently, a polymethylmethacrylate (PMMA) cement spacer is implanted at the site of the bone defects. At the second stage, when the PMMA-induced membrane is formed 6-8 weeks later, the cement spacer is carefully removed in order to not disturb the induced membrane and the bone graft is performed to fill the bone defects. Although this technique has resulted in satisfactory outcomes in the reconstruction of bone defects, the PMMA spacer used to induce membrane is not degradable and requires surgical removal. In recent years, calcium sulfate has been used as a localized antibiotic delivery vehicle and bone substitute due to its superiorities over PMMA, particularly its completely degradable nature. The present study identified that calcium sulfate can also induce the formation of a membrane. In addition, we hypothesized that the degradability of calcium sulfate may allow one-stage reconstruction of bone defects. The current study presents a clinical case report and review of the literature.
\end{abstract}

Correspondence to: Professor Bin Yu, Department of Orthopaedics and Traumatology, Nanfang Hospital, Southern Medical University, 1838 Guangzhou Avenue North, Guangzhou 510515, P.R. China

E-mail: smuyubin@163.com

*Contributed equally

Abbreviations: PMMA, polymethylmethacrylate; PRP, platelet-rich plasma; CT, computed tomography; MSCs, mesenchymal stem cells; BMP-2, bone morphogenetic protein 2

Key words: Masquelet technique, calcium sulfate, bone defects, hypothesis, polymethylmethacrylate

\section{Introduction}

The aim of bone defect reconstruction is to regenerate bone loss and restore limb function (1). Several approaches can be adopted in the clinic, including autologous bone graft, vascularized fibular autograft and the Ilizarov bone transfer technique. Autologous bone graft is not recommended for reconstruction of massive bone defects due to its increased risk of resorption (2). Vascularized fibular autograft and the Ilizarov bone transfer technique are the most frequently used methods for reconstruction of extensive bone defects (3-5).

The Masquelet technique is another effective way to repair extensive bone defects. This two-staged method for bone reconstruction was first described by Masquelet et al in 1986 (6). The first-stage surgery includes radical debridement of bone and soft tissues, followed by implantation of a polymethylmethacrylate (PMMA) cement spacer at the site of the bone defects. The cement spacer is expected to obviate invasion of fibrous tissue at the recipient site (mechanical role) and induce the surrounding membrane (biological role) (7). After a duration of 6 to 8 weeks, a PMMA-induced pseudosynovial membrane formed. At the second-stage surgery, the cement spacer is removed carefully in order to maintain the already formed membrane and cause minimal disturbance. Following this, the defect is filled with morcellised cancellous autologous bone graft. Masquelet et al (6) reported that this method could repair a bone defect of $25 \mathrm{~cm}$ in length.

Since the first study, the Masquelet technique has been widely used to resolve massive bone defects caused by different diseases and achieved clinical efficacy (8-12). However, efficacy in the cases managed by the Masquelet technique does not mean that this approach is optimal. The main disadvantage of this technique is the PMMA cement spacer, which is not degradable and requires a second surgery for removal. Additionally, the variable antibiotic elution rates of PMMA may affect the antibacterial effect. Furthermore, local implantation of PMMA may lead to a rise in temperature, which limits its combination with heat-sensitive antibiotics (13).

In recent years, calcium sulfate has been used clinically as a delivery vehicle and bone substitute due to its superiorities over PMMA. Firstly, calcium sulfate can be totally degraded, entirely with its antibiotic load. Secondly, the rise in 
temperature is minimal when using calcium sulfate combined with antibiotics. Finally, no residual foreign body remains once the antibiotics have been eluted, reducing the risk of biofilm infections (13).

The present study assessed whether if PMMA is replaced by calcium sulfate as a cement spacer in the Masquelet technique, reconstruction of bone defects may be achieved through one-stage surgery. We hypothesize that this one-stage surgery may consist of three essential steps. The first step is radical debridement of bone and surrounding soft tissues. The second step is mixing calcium sulfate, with or without morcellised cancellous autologous bone or other bone substitutes, and optional additives at a correct ratio. Additives include, but are not limited to, antibiotics, platelet-rich plasma (PRP) and mesenchymal stem cells (MSCs). The last step is stabilization of the bone with appropriate types of fixations.

\section{Case report}

Calcium sulfate can induce the formation of membrane. A 24-year-old male had a main complaint of pain with limited activity of the bilateral feet subsequent to falling down from a height of $\sim 12 \mathrm{~m}$. Physical examinations and radiology tests proved that the patient had multiple fractures all over the body. The present report only focuses on the clinical management of the right foot where an open fracture of the calcaneus was identified (type II of the Gustilo-Anderson classification; with a wound in the planta without loss of soft tissue), closed fractures of the talus neck (type IV of the Hawkins classification), navicular and cuboides, and dislocations of the calcaneocuboid and talonavicular joints (Fig. 1). The patient initially received debridement for the calcaneus with limited internal fixations for the fractures of the talus, navicular and cuboides, as well as dislocated joints (Fig. 2). Postoperatively, aggravating exudation with suppuration occurred at the calcaneal wound and diagnosis of calcaneal infection was established accordingly. One month after the first surgery, the patient underwent pin removal and once more radical debridement of the right calcaneus. Subsequently, local calcium sulfate (Stimulan Rapid Cure; Biocomposites, Ltd., Staffordshire, UK) with vancomycin was implanted at the defect site of the calcaneus (Fig. 3). Approximately 8 weeks after the second surgery, the patient returned for further treatment of the bone defect. X-rays of the right foot clearly showed formation of an induced membrane around the calcaneus (Fig. 4). A computed tomography scan (Fig. 5) also confirmed the existence of the membrane. A one-stage strategy was not performed in this patient due to the following considerations: i) A two-stage surgery was originally planned, therefore, the defect was filled with only calcium sulfate and antibiotics. Considering the large bone defects, we believed that it would be more appropriate to reconstruct the defect with bone graft. ii) The patient preferred the reconstruction of the bone defect with autogenous bone graft. Finally, the calcium sulfate was carefully removed and the defect was filled with autogenous iliac crest. Postoperatively, there was no recurrence of local infection. However, due to the severe injury, although the wound recovered well, there remained deformities, such as the loss of Bohler and Gissane angles and decreased calcaneal height at one year follow-up time (Fig. 6).

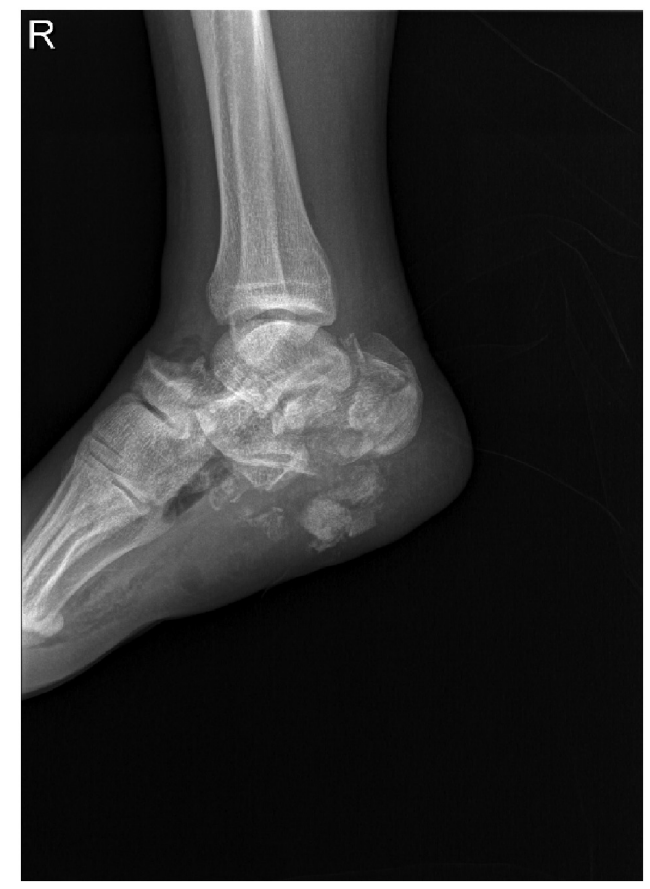

Figure 1. Lateral radiograph of the right foot following trauma. X-ray shows the fractures of the right calcaneus, navicular and cuboides, and dislocations of the calcaneocuboid and talonavicular joints.

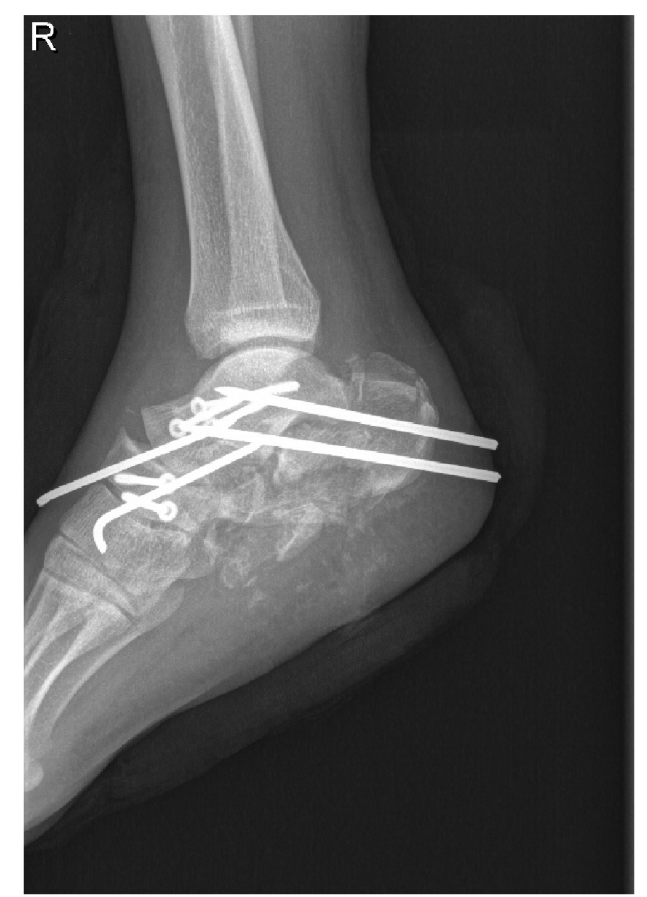

Figure 2. Lateral radiograph of the right foot following the first surgery. $\mathrm{X}$-ray shows the internal fixations for the fractures and dislocated joints. The radiography also revealed an extensive bone defect of the right calcaneus.

Possibility of one-stage surgery. The evidence for the possibility of one-stage surgery included the following aspects. i) Unique biological superiority of calcium sulfate. The advantages of calcium sulfate-based cement include biodegradability, biocompatibility, osteoconductivity and non-exothermic setting reactions (14), of which total biodegradability is the most important as it provides a vital foundation for one-stage 


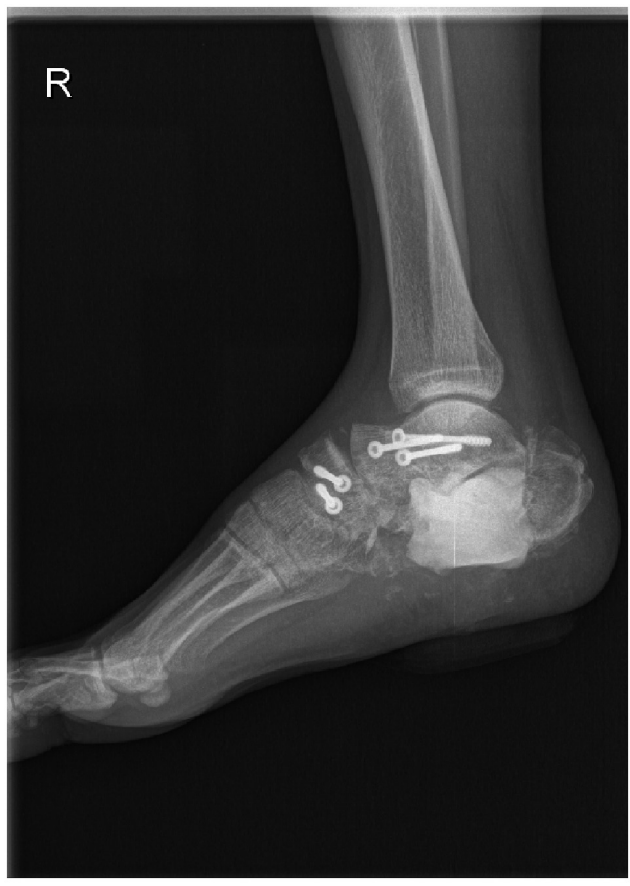

Figure 3. Lateral radiograph of the right foot following the second surgery. X-ray shows the removal of the pins and implanted calcium sulfate at the bone defect site.

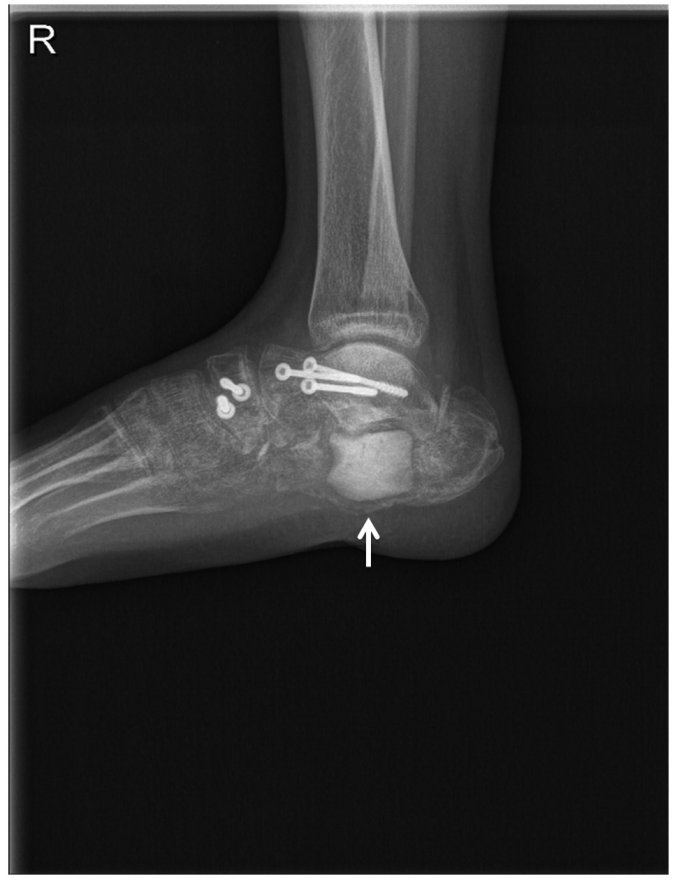

Figure 4. Lateral radiograph of the right foot 8 weeks after the second surgery. X-ray shows the formation of the membrane around the calcium sulfate (arrow).

surgery. ii) Good clinical efficacy of calcium sulfate as a bone substitute. In a previous study regarding the use of calcium sulfate as a bone graft substitute to repair bone defects ranging from 13-138 $\mathrm{cm}^{3}$, Kumar et al (15) concluded that calcium sulfate is a safe, efficient and easily available bone graft substitute for the treatment of osseous defects. Similarly, in a 3-year randomized controlled trial, Di Alberti et al (16) also reported

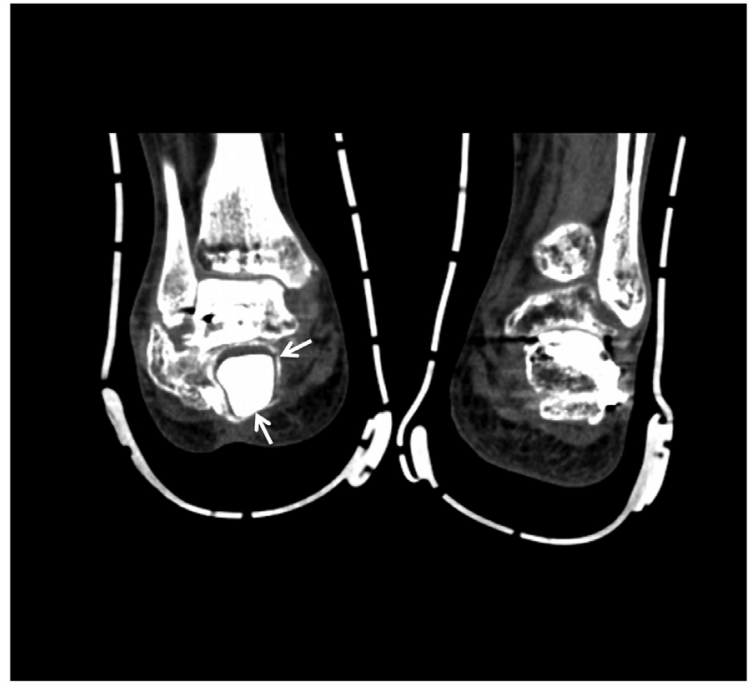

Figure 5. CT scan of the bilateral feet at the coronal plane. This CT scan slide showed the formation of the induced membrane around the calcium sulfate (arrows). CT, computed tomography.

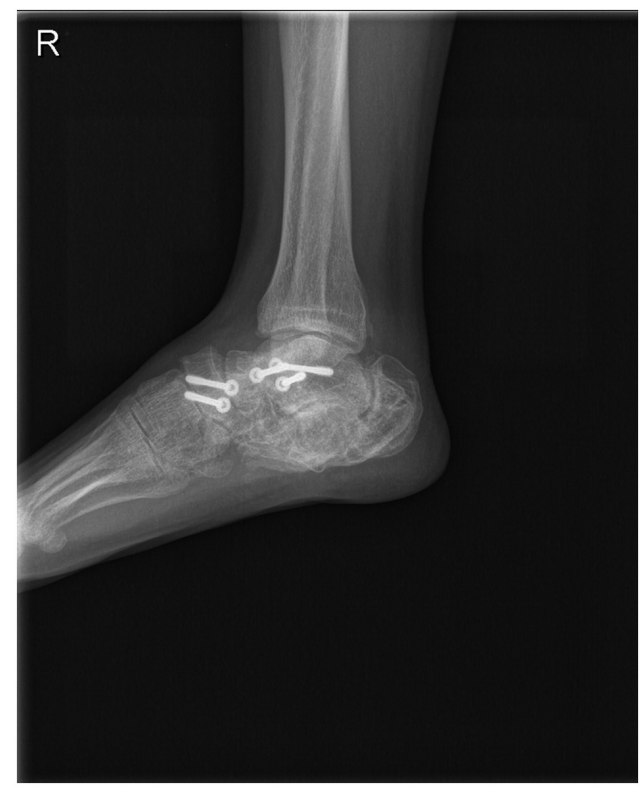

Figure 6. Lateral radiograph of the right foot one year after bone graft using autogenous iliac crest. X-ray shows the effectiveness of the bone defect reconstruction, although sequelae remained in the right cancaneus.

good outcomes of calcium sulfate as a carrier for regeneration of human bone defects. Whether autologous bone or other bone substitutes should be used or not depends on the defect size. If a bone defect is limited, only calcium sulfate may be enough for reconstruction. When a bone defect is extensive, autologous bone or other bone substitutes [such as recombinant human bone morphogenetic protein 2 (rhBMP-2)] can be used in combination with calcium sulfate (17). Of note, radical debridement of the bone and surrounding soft tissues is the premise of the following surgical procedures. iii) With regards to the definitive outcomes of the combination of calcium sulfate with additives in reconstruction of bone defects, Howlin et al (18) identified that calcium sulfate with antibiotics may have a potential ability to reduce or eliminate biofilm 
formation on adjacent periprosthetic tissue and prosthesis material, thus reducing the rates of periprosthetic infection. Following the comparison of PMMA with calcium sulfate as antibiotic carriers at the infected sites, McConoughey et al (13) indicated that calcium sulfate led to similar or improved outcomes compared to PMMA in the inhibition of bacterial growth. In a pilot study regarding the assessment of clinical outcomes of grafted sockets using either calcium sulfate-PRP or calcium sulfate alone in the socket preservation procedure, Cheah et al (19) reported that calcium sulfate-PRP-grafted sites had a higher mineralized bone content compared to the calcium sulfate-grafted sites. In a rat experiment, He et al (20) found that the combination of injectable nano calcium sulfate/alginate paste and BMP2-gene-modified MSCs may be a new and effective strategy for repair of bone defects.

\section{Discussion}

Although the Masquelet technique was initially designed for the treatment of bone defects caused by infection, it has now been widely used for the reconstruction of bone defects caused by other disorders. Gouron et al (8) and Pannier et al (9) reported its use in the treatment of congenital pseudarthrosis of the tibia, which resulted in satisfactory bony union. Villemagne et al (10) adopted this method to reconstruct the long bone defect following resection of the malignant bone tumor, indicating that this simple and reliable technique may lead to promising clinical outcomes.

Previous studies have reported its application mainly for the defects in the long bones. Recently, this technique was also used for repair defects in the foot and hand. Makridis et al (21) used this technique to repair an extensive defect of the first metatarsal bone in a 53-year-old man, who was able to conduct normal daily activities with no pain 18 months postoperatively. Flamans et al (22) used this approach for hand and wrist bone defects in 11 cases, 9 of which achieved bone union with no septic complications at the 1-year follow up.

Recently, investigations addressing the similarities and differences between PMMA and calcium sulfate as two types of cement spacer have drawn wide attention. In an in vitro study, McConoughey et al (13) found that calcium sulfate beads achieved similar or a more improved efficacy compared to the PMMA beads in inhibiting bacterial growth. Grimsrud et al (23) reported similar characteristics of in vitro elution between voriconazole-loaded PMMA bone cement and calcium sulfate bone substitute. A prospective, randomized clinical trial (24), which compared the clinical efficacy of antibiotic-impregnated calcium sulfate with antibiotic-impregnated PMMA for the treatment of chronic osteomyelitis and infected nonunion, revealed that calcium sulfate was equivalent to PMMA in efficacy of eradicating infection, but required fewer subsequent surgical procedures. In a biomechanical pull-out strength test regarding the two cement spacers used for augmentation of a failed pedicle screw, Güler et al (25) found that calcium sulfate augmentation may not be as strong as PMMA augmentation.

Taken together, the previous and present studies have confirmed that calcium sulfate, as a novel delivery vehicle, possesses similar effectiveness as PMMA, but a clear characteristic of total biodegradability, which highlights its superiority over PMMA. However, it should be noted that calcium sulfate is less strong than PMMA.

Currently, the differences between the PMMA-induced membrane and calcium sulfate-induced membrane remain to be elucidated. However, it is clear that the PMMA-induced psedosynovial membrane is characterized by maturing vascularized fibrous tissue (26), which secretes several growth factors, including the vascular endothelial growth factor, BMP-2 and transforming growth factor- $\beta 1$. Whether the calcium sulfate-induced membrane has similar characteristics requires further studies. In addition, detailed differences between the PMMA- and calcium sulfate-induced membranes should be further explored.

Although in the present study, calcium sulfate was able to induce the formation of membrane, numerous aspects remain to be elucidated, such as changes to the induced membrane and the generated space during the degradation process and following degradation. In addition, the fluid is produced around the spacer during the resorption of calcium sulfate; however, compositions of the fluid as well as its effects on the surrounding bones and soft tissues remain to be elucidated. Therefore, more studies should be performed to improve the understanding of calcium sulfate and the induced membrane.

In the present study concerning the repair of bone defects, calcium sulfate was demonstrated to induce the formation of a membrane similar to PMMA and we hypothesized that one-stage surgery may be achieved to reconstruct bone defects with calcium sulfate as a cement spacer. Future studies may develop the use of this one-stage hypothesis.

\section{Acknowledgements}

The authors are grateful for the support of the National Natural Science Foundation of China (grant no. 81572165).

\section{References}

1. Giannoudis PV, Faour O, Goff T, Kanakaris N and Dimitriou R: Masquelet technique for the treatment of bone defects: Tips-tricks and future directions. Injury 42: 591-598, 2011.

2. Weiland AJ, Phillips TW and Randolph MA: Bone grafts: A radiologic, histologic, and biomechanical model comparing autografts, allografts, and free vascularized bone grafts. Plast Reconstr Surg 74: 368-379, 1984.

3. Rigal S, Merloz P, Le Nen D, Mathevon H and Masquelet AC; French Society of Orthopaedic Surgery and Traumatology (SoFCOT): Bone transport techniques in posttraumatic bone defects. Orthop Traumatol Surg Res 98: 103-108, 2012.

4. Pederson WC and Person DW: Long bone reconstruction with vascularized bone grafts. Orthop Clin North Am 38: 23-35, 2007.

5. Tu Y, Ueng SW, Yeh W, Yen C and Wang K: Reconstruction of post-traumatic long bone defect with vascularized bone graft. J Orthop Trauma 14: 138, 2000.

6. Masquelet AC, Fitoussi F, Begue T and Muller GP: Reconstruction of the long bones by the induced membrane and spongy autograft. Ann Chir Plast Esthet 45: 346-353, 2000 (In French).

7. Pelissier P, Masquelet AC, Bareille R, Pelissier SM and Amedee J: Induced membranes secrete growth factors including vascular and osteoinductive factors and could stimulate bone regeneration. J Orthop Res 22: 73-79, 2004.

8. Gouron R, Deroussen F, Juvet M, Ursu C, Plancq MC and Collet LM: Early resection of congenital pseudarthrosis of the tibia and successful reconstruction using the Masquelet technique. J Bone Joint Surg Br 93: 552-554, 2011.

9. Pannier S, Pejin Z, Dana C, Masquelet AC and Glorion C: Induced membrane technique for the treatment of congenital pseudarthrosis of the tibia: Preliminary results of five cases. J Child Orthop 7: 477-485, 2013. 
10. Villemagne T, Bonnard C, Accadbled F, L'kaissi M, de Billy B and Sales de Gauzy J: Intercalary segmental reconstruction of long bones after malignant bone tumor resection using primary methyl methacrylate cement spacer interposition and secondary bone grafting: The induced membrane technique. J Pediatr Orthop 31: 570-576, 2011.

11. Wong TM, Lau TW, Li X, Fang C, Yeung K and Leung F: Masquelet technique for treatment of posttraumatic bone defects. ScientificWorldJournal 2014: 710302, 2014.

12. Karger C, Kishi T, Schneider L, Fitoussi F and Masquelet AC; French Society of Orthopaedic Surgery and Traumatology (SoFCOT): Treatment of posttraumatic bone defects by the induced membrane technique. Orthop Traumatol Surg Res 98: 97-102, 2012.

13. McConoughey SJ, Howlin RP, Wiseman J, Stoodley P and Calhoun JH: Comparing PMMA and calcium sulfate as carriers for the local delivery of antibiotics to infected surgical sites. J Biomed Mater Res B Appl Biomater 103: 870-877, 2015.

14. Koh I, López A, Helgason B and Ferguson SJ: The compressive modulus and strength of saturated calcium sulphate dihydrate cements: Implications for testing standards. J Mech Behav Biomed Mater 34: 187-198, 2014.

15. Kumar CY, Nalini KB, Menon J, Patro DK and BH: Calcium sulfate as bone graft substitute in the treatment of osseous bone defects, a prospective study. J Clin Diagn Res 7: 2926-2928, 2013

16. Di Alberti L, Tamborrino F, Lo Muzio L, D'Agostino A Trevisiol L, De Santis D, Nocini PF and Bertossi D: Calcium sulfate barrier for regeneration of human bone defects. 3 years randomized controlled study. Minerva Stomatol: Jun 11, 2013 (Epub ahead of print).

17. Chen H, Cui X, Yu X, Tian X, Zhang B, Tang P and Wang Y: Effects of chitosan-coated pressed calcium sulfate pellets combined with recombinant human bone morphogenetic protein 2 on bone formation in femoral condyle-contained bone defects. J Craniofac Surg 21: 188-197, 2010.

18. Howlin RP, Brayford MJ, Webb JS, Cooper JJ, Aiken SS and Stoodley P: Antibiotic-loaded synthetic calcium sulfate beads for prevention of bacterial colonization and biofilm formation in periprosthetic infections. Antimicrob Agents Chemother 59: 111-120, 2015.
19. Cheah CW, Vaithilingam RD, Siar CH, Swaminathan D and Hornbuckle GC: Histologic, histomorphometric, and cone-beam computerized tomography analyses of calcium sulfate and platelet-rich plasma in socket preservation: A pilot study. Implant Dent 23: 593-601, 2014

20. He X, Dziak R, Mao K, Genco R, Swihart M, Li C and Yang S: Integration of a novel injectable nano calcium sulfate/alginate scaffold and BMP2 gene-modified mesenchymal stem cells for bone regeneration. Tissue Eng Part A 19: 508-518, 2013.

21. Makridis KG, Theocharakis S, Fragkakis EM and Giannoudis PV: Reconstruction of an extensive soft tissue and bone defect of the first metatarsal with the use of Masquelet technique: A case report. Foot Ankle Surg 20: e19-e22, 2014.

22. Flamans B, Pauchot J, Petite H, Blanchet N, Rochet S, Garbuio P, Tropet Y and Obert L: Use of the induced membrane technique for the treatment of bone defects in the hand or wrist, in emergency. Chir Main 29: 307-314, 2010 (In French).

23. Grimsrud C, Raven R, Fothergill AW and Kim HT: The in vitro elution characteristics of antifungal-loaded PMMA bone cement and calcium sulfate bone substitute. Orthopedics 34: e378-e381, 2011.

24. McKee MD, Li-Bland EA, Wild LM and Schemitsch EH: A prospective, randomized clinical trial comparing an antibioticimpregnated bioabsorbable bone substitute with standard antibiotic-impregnated cement beads in the treatment of chronic osteomyelitis and infected nonunion. J Orthop Trauma 24: 483-490, 2010.

25. Güler UO, Derincek A, Hersekli MA, Ozalay M, Cinar BM and Acaroğlu E: Restoration of pull-out strength of the failed pedicle screw: Biomechanical comparison of calcium sulfate vs polymethylmethacrylate augmentation. Acta Orthop Traumatol Turc 48: 202-206, 2014.

26. Aho OM, Lehenkari P, Ristiniemi J, Lehtonen S, Risteli J and Leskelä HV: The mechanism of action of induced membranes in bone repair. J Bone Joint Surg Am 95: 597-604, 2013. 